\title{
On the Koebe quarter theorem for polynomials
}

\author{
Jimmy Dillies, Dmitriy Dmitrishin, Andrey Smorodin, \\ Alex Stokolos
}

\begin{abstract}
The Koebe One Quarter Theorem states that the range of any Schlicht function contains the centered disc of radius $1 / 4$ which is sharp due to the value of the Koebe function at -1 . A natural question is finding polynomials that set the sharpness of the Koebe Quarter Theorem for polynomials. In particular, it was asked in [8] whether Suffridge polynomials [15] are optimal. For polynomials of degree 1 and 2 that is obviously true. It was demonstrated in [10] that Suffridge polynomials of degree 3 are not optimal and a promising alternative family of polynomials was introduced. These very polynomials were actually discovered earlier independently by M. Brandt [4] and D. Dimitrov [7]. In the current article we reintroduce these polynomials in a natural way and make a far-reaching conjecture that we verify for polynomials up to degree 6 and with computer aided proof up to degree 51 . We then discuss the ensuing estimates for the value of the Koebe radius for polynomials of a specific degree.
\end{abstract}

Анотація. Теорема Кебе про одну чверть стверджує, що область зна-
чень будь-якої шліхт функцї містить диск радіусу $1 / 4$ з центром в точці
$z=0$ Ц $о$ оцінка на радіус є точною і досягається для значення функ-
ції Кебе в точці $z=-1$. Природним питанням є пошук поліномів, які
визначають остаточність теореми Кебе. Зокрема, Димитров ставив пи-
тання, чи є оптимальними поліноми Саффріджа. Для поліномів степеня
1 та 2 це, очевидно, так. Дмитришин, Дьяконов і Стоколос, у спільній
роботі показали, що поліноми Саффріджа степеня 3 не є оптимальними,
і запропонували перспективне альтернативне сімейство поліномів. Ви-
явилося, що ці поліноми були відкриті раніше Брандтом і, незалежно,
Димитровим. В даній статті такі поліноми означено більш природним
чином. Крім того в роботі висунуто важливу гіпотезу, яку перевірено
для поліномів степеня $\leqslant 6$ вручну, а за допомогою комп'ютерного пакета

2010 Mathematics Subject Classification: 30C10, 30C25, 30C55, 30C75

Keywords: Koebe one-quarter theorem, Koebe radius, univalent polynomial

Ключові слова: теорема Кебе про чверть, радіус Кебе, унівалентний многочлен DOI: http://dx.doi.org/10.15673/tmgc.v14i3.2057 
Wolfram Mathematica - для поліномів степеня $\leqslant 51$. Ми також обговорюємо оцінки значення радіусу Кебе для деяких поліномів.

\section{INTRODUCTION}

Geometric complex analysis has arisen from two fundamental statements: the Koebe Quarter Theorem and the Bieberbach Conjecture. Koebe's theorem states that for any function $f \in \mathcal{S}$ the image $f(\mathbb{D})$ contains a disc of radius $1 / 4$, where $\mathbb{D}=\{|z|<1\}$ and

$$
\mathcal{S}=\left\{f(z): f(0)=0, f^{\prime}(0)=1, f(z) \text { is univalent in } \mathbb{D}\right\} .
$$

The Bieberbach Conjecture says that $\left|a_{k}\right| \leqslant k$ for all $k=1,2, \ldots$ where $f \in \mathcal{S}$ and the $a_{k}$ are the Taylor coefficients of $f$. For many decades this conjecture was a driving force of the development of geometric complex analysis. Many outstanding mathematicians contributed through partial solutions until it was resolved in full generality by Louis de Brange in 1984 .

Both theorems offer sharp bounds as the so-called Koebe function

$$
\mathcal{K}(z):=\frac{z}{(1-z)^{2}}=z+2 z^{2}+3 z^{3}+\cdots, \quad z \in \mathbb{D},
$$

is an extremizer for both statements. One can see that the radius $\frac{1}{4}$ in Koebe's theorem is optimal, as also is the estimate $\left|a_{k}\right| \leqslant k$ in de Brange's result. A natural question is whether the constant $1 / 4$ as well as the estimate $\left|a_{k}\right| \leqslant k$ can be improved for polynomials of a specific degree and what would be a polynomial analogue of the Koebe function. Say, for polynomials of the first degree the constant is trivially 1; a simple computation demonstrates that for polynomials of degree 2 it is $1 / 2$. The task was formalized by Dimitrov who asked:

Problem 1.1. [8, Problem 5] For any $N \in \mathbb{Z}_{+}$, find a polynomial

$$
p_{N}(z)=z+a_{2} z^{2}+\ldots+a_{N} z^{n} \in \mathcal{S}
$$

for which the infimum $r_{N}:=\inf \left\{\left|p_{N}(z)\right|: z=e^{i t}, 0 \leqslant t \leqslant 2 \pi\right\}$ is attained.

Let us call $r_{N}$ the Koebe radius. Obviously, we have $r_{N} \geqslant 1 / 4$. In this article a new interesting family of typically real polynomials is introduced. We conjecture that they are univalent (this is proven for degree $\leqslant 6$ ) and that they attain the value of the Koebe radius.

The following statement a central in the paper:

Conjecture 1.2. The value of the Koebe radius for the polynomials of degree $N$ is

$$
\frac{1}{4} \sec ^{2} \frac{\pi}{N+2} .
$$


One cannot emphasize enough the relation between this work and earlier work of Dimitrov. In particular, in [9], the author established a relation between Egerváry-Szász and Suffridge polynomials. Dimitrov's main result was actually hinted at by the surprising link between Suffridge polynomials and Fejér's kernel. His so-called Féjer polynomials [9, Eq. (3.3)] correspond to our Egerváry-Szász polynomials, $E_{N}(t)$. So in a sense, our work parallels that of Dimitrov even though our initial motivations were different.

\section{SUFFridge POLYNOMIALS}

A natural approach to Dimitrov's problem would be to look at truncations of the Koebe function. However, there is a significant difference between extremal analytic functions and polynomials. Since the derivative of a function univalent in $\mathbb{D}$ has roots outside $\mathbb{D}$, Vieta's theorem implies the estimate on the leading coefficient

$$
\left|a_{N}\right| \leqslant \frac{1}{N}
$$

Since the coefficients of the Koebe function increase, the truncation is not a univalent in $\mathbb{D}$ polynomial.

Unfortunately, the variety of known polynomials univalent in $\mathbb{D}$ is quite limited. The estimate (2.1) suggests considering the polynomials

$$
A_{N}(z)=\sum_{k=1}^{N} \frac{1}{k} z^{k} .
$$

These are partial sums of the function $-\log (1-z)$ which is univalent in $\mathbb{D}$. They were proven to be univalent in $\mathbb{D}$ by $\mathrm{G}$. W. Alexander in the milestone paper [1]. For these polynomials, $\left|A_{N}(-1)\right| \geqslant \frac{1}{2}$ and $\frac{1}{2}$ is sharp.

Other popular extremal polynomials satisfying (2.1) are the Fejér polynomials

$$
F_{N}(z)=\sum_{k=1}^{N}\left(1-\frac{k-1}{N}\right) z^{k} .
$$

These again indicate that the constant $\frac{1}{2}$ might be sharp in general. Certainly, we need more polynomials to test. However, to construct new extremal univalent polynomials is a quite challenging task.

Returning to the Koebe function, we should recall that it is extremal for the Bieberbach conjecture and has increasing coefficients, while the coefficients in the above examples are decreasing. A powerful idea of Ted Suffridge [15] was to multiply the Fejér coefficients by the sine factor $\sin \frac{\pi k}{N+1}$, making the new coefficients increase up to some level. He introduced a 
remarkable family of extremal polynomials

$$
S_{N, j}(z)=\sum_{k=1}^{N}\left(1-\frac{k-1}{N}\right) \frac{\sin (\pi k j /(N+1))}{\sin (\pi j /(N+1))} z^{k}, \quad j=1, \ldots, N,
$$

which turn (2.1) into equality. He proved that they are univalent in $\mathbb{D}$. Below instead of $S_{N, 1}(z)$ we will simply write $S_{N}(z)$.

Also, Suffridge showed that whenever $p_{N}(z)$ is a polynomial in $\mathcal{S}$ with real coefficients and $\left|a_{N}\right|=1 / N$, the remaining coefficients of $p_{N}(z)$ are also dominated by the coefficients of $S_{N}(z)$.

Moreover,

$$
\left|S_{N}(-1)\right|=\frac{1}{4} \frac{N+1}{N} \sec ^{2} \frac{\pi}{2(N+1)} \rightarrow \frac{1}{4},
$$

hence these polynomials indicate that $1 / 4$ is asymptotically sharp for the polynomial version of the Koebe Quarter Theorem (cf. [6]). Thus, Suffridge polynomials may be considered as a counterpart of the Koebe function.

Note that the value $\left|S_{N}(-1)\right|$ is the smallest distance from the image of the unit circle to the origin for polynomials $S_{N}(z)$, but only for even degree. For polynomials of odd degree the infimum $\inf \left\{\left|S_{N}(z)\right|:|z|=1\right\}$ is not achieved at $z=-1$, but at a different point $\xi$ such that $S_{N}^{\prime}(\xi)=0[10]$ (see Fig. 1).
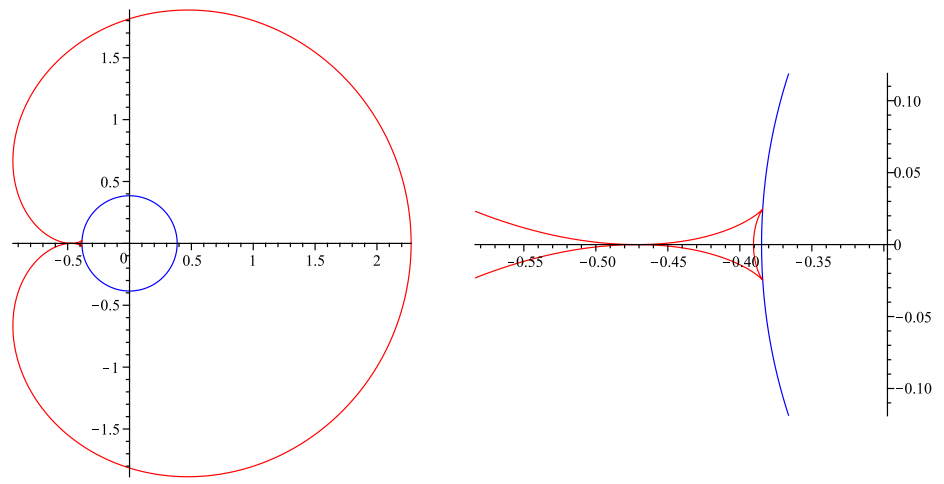

Fig 1: The image and fragment for $S_{3}(\mathbb{D})$.

Also, note that the roots of the derivative of a Suffridge polynomial are on $\partial \mathbb{D}$, and the leading coefficient is $1 / N$ - the extremal case of the univalence property. What could be a better candidate to be a solution to Dimitrov's problem? Actually, Dimitrov [8, p. 16] asked a specific question about the Suffridge polynomial $S_{N}(z)$ : Is it extremal for every fixed $N$ ? Note that it is indeed extremal for $N=1,2$.

Later in this work we will provide evidence that the range of Brandt polynomials contain a central disc of radius $\frac{1}{4} \sec ^{2}(\pi / N+2)$ by working out explicit cases. Our conjecture is that this holds for all $N$ which would allow 
Brandt polynomials to be used in future work as probes to text extremal constructions.

\section{NEW POLYNOMIALS}

We have analyzed the way the Suffridge polynomials emerged. They came out as multiplier operators with some sine multipliers applied to the Fejér polynomials. In turn, the Fejér polynomials arose as a solution of the following extremal problem.

Let $f_{N}(t)=1+a_{1} \cos t+\cdots+a_{N} \cos N t \geqslant 0$. Then $f_{N}(t) \leqslant N+1$ and the Fejér polynomials are extremal here. Their coefficients can be computed from the Fejér-Riesz representation

$$
\Phi_{N}(t)=\frac{1}{N+1}\left|\sum_{k=0}^{N} e^{i k t}\right|^{2}=1+2 \sum_{k=1}^{N}\left(1-\frac{k}{N+1}\right) \cos k t .
$$

Furthermore, for the trigonometric polynomial

$$
F_{N}(t)=1+2 \sum_{k=1}^{N} a_{k} \cos k t
$$

the following Fejér inequality is valid:

$$
\left|a_{1}\right| \leqslant 2 \cos \frac{\pi}{N+2}
$$

and here the extremal polynomials are the Egérvary-Szász polynomials [17]

$$
E_{N}(t)=\frac{N+2}{2}+\sum_{k=1}^{N}\left((N+1-k) \cos \frac{k \pi}{N+2}+\frac{\sin \frac{\pi(k+1)}{N+2}}{\sin \frac{\pi}{N+2}}\right) \cos k t .
$$

The expression $\frac{2}{N+2} E_{N}(t)$ can be written in the following form [5]:

$$
\frac{2}{N+2}\left|\sum_{k=0}^{N} \sin \frac{\pi(k+1)}{N+2} e^{i k t}\right|^{2}=\sum_{k=0}^{N} b_{k} \cos k t
$$

where $b_{0}=1$ and for $k=1, \ldots, N$,

$$
b_{k}=\frac{(N-k+3) \sin \frac{(k+1) \pi}{N+2}-(N-k+1) \sin \frac{(k-1) \pi}{N+2}}{(N+2) \sin \frac{\pi}{N+2}} .
$$

Now, let us apply the same approach to the Egerváry-Szász polynomials, i.e. multiply the coefficients $(3.1)$ by $\sin \frac{k \pi}{N+1}$ and introduce the new polynomials

$$
P_{N}(z)=\csc \frac{2 \pi}{N+2} \sum_{k=1}^{N} b_{k} \sin \frac{\pi k}{N+2} z^{k}
$$


Below are some examples:

$$
\begin{aligned}
& P_{1}(z)=z, \quad P_{2}(z)=z+\frac{1}{2} z^{2}, \\
& P_{3}(z)=z+\frac{2}{\sqrt{5}} z^{2}+\frac{1}{2}\left(1-\frac{1}{\sqrt{5}}\right) z^{3} \\
& P_{4}(z)=z+\frac{7}{6} z^{2}+\frac{2}{3} z^{3}+\frac{1}{6} z^{4}, \\
& P_{5}(z)=z+\frac{8-40 \cos ^{2}\left(\frac{\pi}{7}\right)+32 \cos ^{3}\left(\frac{\pi}{7}\right)-24 \cos \left(\frac{\pi}{7}\right)}{40 \cos ^{3}\left(\frac{\pi}{7}\right)-30 \cos \left(\frac{\pi}{7}\right)-32 \cos ^{2}\left(\frac{\pi}{7}\right)+7} z^{2}+ \\
& +\frac{24 \cos ^{3}\left(\frac{\pi}{7}\right)-28 \cos ^{2}\left(\frac{\pi}{7}\right)-18 \cos \left(\frac{\pi}{7}\right)+4}{40 \cos ^{3}\left(\frac{\pi}{7}\right)-30 \cos \left(\frac{\pi}{7}\right)-32 \cos ^{2}\left(\frac{\pi}{7}\right)+7} z^{3}+ \\
& +\frac{16 \cos ^{3}\left(\frac{\pi}{7}\right)-16 \cos ^{2}\left(\frac{\pi}{7}\right)-12 \cos \left(\frac{\pi}{7}\right)+4}{40 \cos ^{3}\left(\frac{\pi}{7}\right)-30 \cos \left(\frac{\pi}{7}\right)-32 \cos ^{2}\left(\frac{\pi}{7}\right)+7} z^{4}+ \\
& +\frac{8 \cos ^{3}\left(\frac{\pi}{7}\right)-4 \cos ^{2}\left(\frac{\pi}{7}\right)-6 \cos \left(\frac{\pi}{7}\right)+1}{40 \cos ^{3}\left(\frac{\pi}{7}\right)-30 \cos \left(\frac{\pi}{7}\right)-32 \cos ^{2}\left(\frac{\pi}{7}\right)+7} z^{5}, \\
& P_{6}(z)=z+\frac{9+8 \sqrt{2}}{4 \sqrt{2}+8} z^{2}+\frac{6 \sqrt{2}+10}{4 \sqrt{2}+8} z^{3}+\frac{4 \sqrt{2}+6}{4 \sqrt{2}+8} z^{4}+\frac{2 \sqrt{2}+2}{4 \sqrt{2}+8} z^{5}+\frac{1}{4 \sqrt{2}+8} z^{6} .
\end{aligned}
$$

Theorem 3.1. The following representation is valid for $t \in(0, \pi), t \neq \frac{2 \pi}{N+2}$ :

$$
\begin{aligned}
P_{N}\left(e^{i t}\right)= & \frac{1}{2\left(\cos t-\cos \frac{2 \pi}{N+2}\right)}+ \\
& \quad+\frac{1-\cos \frac{2 \pi}{N+2}}{(N+2)(1-\cos t)} \frac{\sin t \sin \frac{N+2}{2} t}{\left(\cos t-\cos \frac{2 \pi}{N+2}\right)^{2}} e^{\frac{N+2}{2} i t} .
\end{aligned}
$$

Proof. We begin with

$$
\begin{aligned}
& P_{N}(z)=\frac{1}{(N+2) \sin \frac{2 \pi}{N+2} \sin \frac{\pi}{N+2}} \times \\
& \quad \times \sum_{k=1}^{N}\left[(N-k+3) \sin \frac{(k+1) \pi}{N+2}-(N-k+1) \sin \frac{(k-1) \pi}{N+2}\right] \sin \frac{k \pi}{N+2} z^{k} .
\end{aligned}
$$

Having in mind that

$$
\left[2 \sin (\pi)-0 \cdot \sin \frac{N \pi}{N+2}\right] \sin \frac{(N+1) \pi}{N+2} z^{N+1} \equiv 0
$$

we can sum up to $N+1$. A further modification produces

$$
P_{N}(z)=\frac{1}{(N+2) \sin \frac{2 \pi}{N+2}} \sum_{k=1}^{N+1}\left[(N-k+2) \sin \frac{2 k \pi}{N+2}+2 \frac{\cos \frac{\pi}{N+2}}{\sin \frac{\pi}{N+2}} \sin ^{2} \frac{k \pi}{N+2}\right] z^{k} .
$$


An important observation is that

$$
\frac{N+1}{N+2} \cdot S_{N+1,2}(z)=\frac{1}{(N+2) \sin \frac{2 \pi}{N+2}} \sum_{k=1}^{N+1}(N-k+2) \sin \frac{2 k \pi}{N+2} \cdot z^{k},
$$

where $S_{N+1,2}(z)$ is the second Suffridge polynomial of order $N+1$. By using formula (5) in [15, p. 496] for $n=N+1$ and $j=2$ we get

$$
\begin{aligned}
\frac{N+1}{N+2} \cdot S_{N+1,2}\left(e^{i t}\right)=\frac{1}{2(\cos t-} & \left.\cos \frac{2 \pi}{N+2}\right) \\
& +\frac{1}{N+2} \cdot \frac{\sin t \cdot \sin \frac{N+2}{2} t}{\left(\cos t-\cos \frac{2 \pi}{N+2}\right)^{2}} \cdot e^{\frac{N+2}{2} i t} .
\end{aligned}
$$

Meanwhile,

$$
\sum_{k=1}^{N+1} \sin ^{2} \frac{k \pi}{N+2} e^{i k t}=\sin ^{2} \frac{\pi}{N+2} \cdot \frac{\sin \frac{N+2}{2} t}{\cos t-\cos \frac{2 \pi}{N+2}} \cdot \frac{\sin t}{1-\cos t} \cdot e^{i \frac{N+2}{2} t} .
$$

By combining both formulas, we get the formula in the theorem.

Note that the right hand side of (3.3) has removable singularities, thus it is in fact a trigonometric polynomial.

Theorem 3.2. The following representation is valid for $t \in(0, \pi), t \neq \frac{2 \pi}{N+2}$ :

$$
\begin{aligned}
& \begin{array}{r}
\left|P_{N}\left(e^{i t}\right)\right|^{2}= \\
=\left(\frac{\cos \frac{N+2}{2} t}{\cos t-\cos \frac{2 \pi}{N+2}}+\frac{2}{N+2} \frac{1-\cos \frac{2 \pi}{N+2}}{1-\cos t} \frac{\sin t}{\left(\cos t-\cos \frac{2 \pi}{N+2}\right)^{2}} \sin \frac{N+2}{2} t\right)^{2}+ \\
+\left(\frac{\sin \frac{N+2}{2} t}{\cos t-\cos \frac{2 \pi}{N+2}}\right)^{2} .
\end{array}
\end{aligned}
$$

Theorem 3.2 can be directly verified by tedious standard computations.

Further, in order to better understand the behaviour of $P_{N}$, we will pull back its norm to $\mathbb{R}_{0}^{+}$via the Weierstrass map. The pull back,

$$
R_{N}(x)=\left.\left|P_{N}\left(e^{i t}\right)\right|^{2}\right|_{t=2 \arctan x}
$$

allows us to study a single period of the function.

Theorem 3.3. If $\left(R_{N}(x)\right)^{\prime}<0$ for all $x \in(0, \infty)$ then $P_{N}(\mathbb{D})$ contains a central disc of radius

$$
\frac{1}{4} \sec ^{2} \frac{\pi}{N+2}
$$


Proof. Taking the imaginary part we get

$$
\Im\left(P_{N}\left(e^{i t}\right)\right)=\frac{1-\cos \frac{2 \pi}{N+2}}{(N+2)(1-\cos t)} \frac{\sin t\left(\sin \frac{N+2}{2} t\right)^{2}}{\left(\cos t-\cos \frac{2 \pi}{N+2}\right)^{2}} .
$$

Since $\Im\left(P_{N}\left(e^{i t}\right)\right) \geqslant 0$ on $[0, \pi]$, the monotonicity of $R_{N}(x)$ in $x$ implies the monotonicity of $\left|P_{N}\left(e^{i t}\right)\right|$ in $t$ which implies that the shortest distance from the boundary $\partial P(\mathbb{D})$ to the origin is at

$$
P_{N}(-1)=\frac{1}{2\left(\cos \pi-\cos \frac{2 \pi}{N+2}\right)}=-\frac{1}{4} \sec ^{2} \frac{\pi}{N+2} .
$$

Theorem efthm3 motivates the following:

Conjecture 3.4. For every polynomial $P_{N}(z)$ the range $P_{N}(\mathbb{D})$ contains a central disc of radius

$$
\frac{1}{4} \sec ^{2} \frac{\pi}{N+2} .
$$

\section{Proof of the conjecture for small $N$}

Our first observation is that $R_{N}(x)$ is of the form

$$
\frac{Q_{N}(x)}{\left(1+x^{2}\right)^{N-1}}
$$

where $Q_{N}$ is an even polynomial of degree $2(N-1)$. Indeed, one shows by induction that $\cos (n \arctan x)$ (or $\sin (n \arctan x))$ is a rational function of the form

$$
\frac{c_{n}(x)}{\left(1+x^{2}\right)^{\frac{n}{2}}}
$$

where $c_{n}$ is a polynomial. An ugly but elementary computation implies then that $R_{N}$ is of the form shown in equation (4.1).

The benefit is that the monotonicity can now checked by a deterministic algorithm: using a Sturm sequence one can count the real roots of the numerator of the derivative of $R_{N}(x)$ :

$$
\left(1+x^{2}\right)^{N-2} \Delta_{N}(x):=\left(1+x^{2}\right)^{N-2}\left(Q_{N}^{\prime}(x)\left(1+x^{2}\right)-2(N-1) x Q_{N}(x)\right) .
$$

This allows us to determine the univalence of the function $P$ :

4.1. The case $N=1$. In this case $P_{1}(z)=z, R_{1}(x)=1$, thus the Koebe radius is 1 .

4.2. The case $N=2$. In this case $Q_{2}(x)=\frac{9+x^{2}}{4}$, and the Koebe radius $r_{2}$ is $\left|P_{2}(-1)\right|=1 / 2$. 
4.3. The case $N=3$. In this case

$$
Q_{3}(x)=\frac{1}{10}\left(27+9 \sqrt{5}+(10 \sqrt{5}-18) x^{2}+(35-15 \sqrt{5}) x^{4}\right)
$$

As $\Delta_{3}$ is quadratic, is easy to check that $R_{3}$ is decreasing. on $\mathbb{R}^{+}$. This implies the estimate $r_{3} \leqslant\left|P_{3}(-1)\right|=\frac{3-\sqrt{5}}{2}=0.382 \ldots$ for the Koebe radius. Note that for the Suffridge polynomial we have $\left|S_{3}(-1)\right|=0.3905 \ldots$ and the minimal distance from the image of the unit circle to the origin is $0.3849 \ldots$. [10]. These estimates imply a negative answer to Dimitrov's question for cubic polynomials.

4.4. The case $N=4$. In this case

$$
Q_{4}(x)=\frac{1}{9}\left(x^{2}+9\right)\left(x^{4}-2 x^{2}+9\right)
$$

(This can also be seen from $\Delta$ which is biquadratic). The discriminant is negative, and therefore the smallest value for $R_{4}(x)$ is at -1 , which implies $r_{4} \leqslant\left|P_{4}(-1)\right|=1 / 3$.

4.5. The case $N=5$. We have that

$$
\begin{aligned}
Q_{5}(x)= & -49 x^{8}\left(121 \sin \frac{\pi}{14}-42\left(3+5 \sin \frac{3 \pi}{14}\right)+55 \cos \frac{\pi}{7}\right)+ \\
& +4 x^{6}\left(8924-9107 \sin \frac{\pi}{14}+15094 \sin \frac{3 \pi}{14}-4507 \cos \frac{\pi}{7}\right)+ \\
& +2 x^{4}\left(84326-20935 \sin \frac{\pi}{14}+116342 \sin \frac{3 \pi}{14}+33443 \cos \frac{\pi}{7}\right)- \\
& -4 x^{2}\left(37328+61431 \sin \left(\frac{\pi}{14}+31802 \sin \frac{3 \pi}{14}+133139 \cos \frac{\pi}{7}\right)+\right. \\
& +21\left(22702+3859 \sin \frac{\pi}{14}+30218 \sin \frac{3 \pi}{14}+31141 \cos \frac{\pi}{7}\right) \times \\
& \times \frac{1}{784\left(\sin \frac{3 \pi}{28}+\cos \frac{3 \pi}{28}\right)^{14}}
\end{aligned}
$$

and

$$
\begin{aligned}
\frac{\Delta}{16 x}= & x^{6}\left(-1375+1589 \sin \frac{\pi}{14}-2402 \sin \frac{3 \pi}{14}+906 \cos \frac{\pi}{7}\right)- \\
& -x^{4}\left(28777+3193 \sin \frac{\pi}{14}+35530 \sin \frac{3 \pi}{14}+23482 \cos \frac{\pi}{7}\right)+ \\
& +x^{2}\left(98155+81679 \sin \frac{\pi}{14}+105874 \sin \frac{3 \pi}{14}+216430 \cos \frac{\pi}{7}\right)- \\
& -5\left(51407+14247 \sin \frac{\pi}{14}+66638 \sin \frac{3 \pi}{14}+78710 \cos \frac{\pi}{7}\right) .
\end{aligned}
$$

Again $P_{5}(z)$ is univalent and this gives us an estimate on the Koebe radius $r_{5} \leqslant\left|P_{5}(-1)\right|=0.3080 \ldots$ 
4.6. The case $N=6$. In this case

$$
\begin{aligned}
Q_{6}(x)= & (6-4 \sqrt{2}) x^{10}+(246-172 \sqrt{2}) x^{8}+4(70 \sqrt{2}-99) x^{6}- \\
& -4(30 \sqrt{2}-61) x^{4}-10(9+2 \sqrt{2}) x^{2}+36 \sqrt{2}+54
\end{aligned}
$$

and

$$
\begin{aligned}
\frac{\Delta}{16 x}= & (19 \sqrt{2}-27) x^{8}+(222-156 \sqrt{2}) x^{6}+ \\
& +(150 \sqrt{2}-240) x^{4}+(106-20 \sqrt{2}) x^{2}+(-45-25 \sqrt{2}) .
\end{aligned}
$$

This is the last situation where we can find the roots exactly and this implies the estimate for the Koebe radius $r_{6} \leqslant\left|P_{6}(-1)\right|=0.2929 \ldots$. We conjecture that the estimates obtained are in fact true values.

4.7. Larger $N$. As mentioned above, by using the Weierstrass transform, univalence follows from the study of the roots of $\Delta_{N}$. We used Mathematica that checks exactly that $\Delta(x)$ has no real roots outside 0 for $N$ up to 51 , and the growth of $R_{N}$ can be checked exactly for any $N$.

\section{Koebe RAdius For Univalent POLYNOMIALS}

When this article was in preparation, the authors learned that these polynomials were discovered earlier and independently by Brandt [4] and Dimitrov [7]. Neither considered the problem of uniqueness and their univalence was proved through two different methodologies by Brandt [4] and by Kayumov and Khammatova [11]. Note that the monotonicity of $\left|P_{N}\left(e^{i t}\right)\right|$ implies that $P_{N}(z)$ takes no value more than once on $\partial \mathbb{D}$, whence the polynomial $P_{N}(z)$ is univalent in $\mathbb{D}$ (cf. $[16,6.45$, p. 201]). This gives a third proof of the univalency of $P_{N}(z)$ for small values of $N$.

Let us thus focus directly on their Koebe radius. Theorem 3.3 implies the following estimate on the Koebe radius for univalent polynomials:

$$
r_{N} \leqslant \frac{1}{4} \sec ^{2} \frac{\pi}{N+2}
$$

This estimate may be complemented by an estimate from below. In 1916 Bieberbach provided the estimate

$$
\left|a_{2}\right| \leqslant 2
$$

for the the second Taylor coefficient of a function from $\mathcal{S}$. This estimate implies the Koebe conjecture by the following beautiful argument. Let $f \in \mathcal{S}, f(z)=z+\alpha_{2} z^{2}+\cdots$ and $\gamma \notin f(\mathbb{D})$. Then

$$
\frac{f(z)}{1-\frac{f(z)}{\gamma}}=f(z)\left(1+\frac{f(z)}{\gamma}+\cdots\right)=\left(z+\alpha_{2} z^{2}+\cdots\right)\left(1+\frac{z}{\gamma}+\cdots\right)=
$$




$$
=z+\left(a_{2}+\frac{1}{\gamma}\right) z^{2}+\cdots .
$$

By the Bieberbach estimate we have $\left|\alpha_{2}+\frac{1}{\gamma}\right| \leqslant 2$, hence $|\gamma| \geqslant \frac{1}{2+\left|\alpha_{2}\right|}$, and again by (5.2) we get $|\gamma| \geqslant \frac{1}{4}$ which is a statement of Koebe Theorem.

The above argument implies that $r_{N} \geqslant \frac{1}{2+\sup \left|a_{2}\right|}$. W. Rogosinski and G. Szegó [13] obtained an estimate for the second coefficient of a typically real polynomial, $\left|a_{2}\right| \leqslant 2 \cos 2 \psi_{N}$, where $\psi_{N}=\pi /(N+3)$ if $N$ is odd, and $\psi_{N}$ is the smallest positive root of the equation

$$
(N+4) \sin (N+2) \psi_{N}+(N+2) \sin (N+4) \psi_{N}=0
$$

if $N$ is even. (Recall that a polynomial $p(z)$ with real coefficients is called typically real in $\mathbb{D}$ if $\Im(p(z)) \Im(z) \geqslant 0$ for $z \in \mathbb{D}$.) Since a univalent polynomial with real coefficients is typically real we thus get an estimate on the Koebe radius for univalent polynomials:

$$
r_{N} \geqslant \frac{1}{4} \sec ^{2} \psi_{N}
$$

An alternative approach to the problem of estimating the functionals in complex domains can be found in $[2,3,12,14]$

\section{ACKNOWLEDGEMENTS}

The authors are grateful to Konstantin Dyakonov and Paul Hagelstein for numerous fruitful discussions, useful comments and observations. They also would like to thank Adhemar Bultheel and Plamen Iliev for their time and interesting exchanges and to thank Jerzy Trzecjak for the help in preparation of manuscript. Also, the authors are grateful to the anonymous referees for their valuable comments and spotting of several typos.

\section{REFERENCES}

[1] J. W. Alexander. Functions which map the interior of the unit circle upon simple regions. Ann. of Math. (2), 17(1):12-22, 1915, doi: 10.2307/2007212.

[2] A. K. Bakhtin, G. P. Bakhtina, Yu. B. Zelinskii. Topological-algebraic structures and geometric methods in complex analysis, volume 73 . Proceedings of the Institute of Mathematics of NAS of Ukraine, Kyiv, 2008.

[3] A. K. Bakhtin, I. V. Denega. Weakened problem on extremal decomposition of the complex plane. Mat. Stud., 51(1):35-40, 2019, doi: 10.15330/ms.51.1.35-40.

[4] M. Brandt. Representation formulas for the class of typically real polynomials. Math. Nachr., 144:29-37, 1989, doi: 10.1002/mana.19891440104.

[5] D. A. Brannan. Coefficient regions for univalent polynomials of small degree. Mathematika, 14:165-169, 1967, doi: 10.1112/S0025579300003764.

[6] Antonio Cordova Y., Stephan Ruscheweyh. On maximal ranges of polynomial spaces in the unit disk. Constr. Approx., 5(3):309-327, 1989, doi: 10.1007/BF01889612.

[7] D. Dimitrov. Private communication. 
[8] Dimitar K. Dimitrov. Extremal positive trigonometric polynomials. In Approximation theory, pages 136-157. DARBA, Sofia, 2002.

[9] Dimitar K. Dimitrov. Extremal univalent polynomials subordinating the Koebe function. East J. Approx., 11(1):47-56, 2005.

[10] Dmitriy Dmitrishin, Konstantin Dyakonov, Alex Stokolos. Univalent polynomials and Koebe's one-quarter theorem. Anal. Math. Phys., 9(3):991-1004, 2019, doi: 10.1007/s13324-019-00305-x.

[11] I. R. Kayumov, D. M. Khammatova. On a conjecture about univalent polynomials. arXiv:2002.12304, 2020.

[12] B. A. Klishchuk, R. R. Salimov. Lower bounds for the volume of the image of a ball. Ukr. Math. Journ., 71(6):774-785, 2019.

[13] W. W. Rogosinski, G. Szegö. Extremum problems for non-negative sine polynomials. Acta Sci. Math. (Szeged), 12:112-124, 1950.

[14] R. R. Salimov, B. A. Klishchuk. An extremal problem for the area of the image of a disk. Zap. Nauchn. Sem. S.-Peterburg. Otdel. Mat. Inst. Steklov. (POMI), 456(Issledovaniya po LineĬnym Operatoram i Teorii Funktsǐ. 45):160-171, 2017, doi: 10.1007/s10958-0184015-6.

[15] T. J. Suffridge. On univalent polynomials. J. London Math. Soc., 44:496-504, 1969, doi: $10.1112 / \mathrm{jlms} / \mathrm{s} 1-44.1 .496$.

[16] E. C. Titchmarsh. The theory of functions. Oxford University Press, Oxford, second edition, 1939.

[17] Eugen v. Egerváry, Otto Szász. Einige Extremalprobleme im Bereiche der trigonometrischen Polynome. Math. Z., 27(1):641-652, 1928, doi: 10.1007/BF01171120.

Received: June 20, 2021, accepted: August 28, 2021.

Jimmy Dillies

Department of Mathematics, University of Georgia, Athens GA, 30602, USA

Email: jimmy.dillies@uga.edu

Dmitriy Dmitrishin

Department of Applied Mathematics, Odessa National Polytechnic University, Odessa, 65044, Ukraine

Email: dmitrishin@opu.ua

Andrey Smorodin

Department of Applied Mathematics, Odessa National Polytechnic University, ODESSA, 65044, UKRAINE

Email: andrey.v.smorodin@gmail.com

Alex Stokolos

Department of Mathematics, Georgia Southern University, Statesboro Ga, 30460, USA

Email: astokolos@georgiasouthern.edu 